\title{
The effects of sustained manual pressure stimulation according to Vojta Therapy on heart rate variability
}

\author{
Jaroslav Opavsky ${ }^{\mathrm{a}}$, Martina Slachtova ${ }^{\mathrm{a}}$, Miroslav Kutin ${ }^{\mathrm{b}, \mathrm{c}}$, Pavel Hok ${ }^{\mathrm{d}}$, Petr Uhlira, Hana Opavska ${ }^{\mathrm{a}}$, Petr Hlustik ${ }^{\mathrm{d}}$
}

Background. The physiotherapeutic technique of Vojta reflex locomotion is often accompanied by various autonomic activity changes and unpleasant sensations. It is unknown whether these effects are specific to Vojta Therapy. Therefore, the aim of this study was to compare changes in cardiac autonomic control after Vojta reflex locomotion stimulation and after an appropriate sham stimulation.

Methods. A total of 28 young healthy adults ( $20.4-25.7$ years) were enrolled in this single-blind randomized crossover study. Participants underwent two modes of 20-minute sustained manual pressure stimulation on the surface of the foot on two separate visits. One mode used manual pressure on the lateral heel, i.e., in a zone employed in the Vojta Therapy (active stimulation). The other mode used pressure on the lateral ankle (control), in an area not included among the active zones used by Vojta Therapy and whose activation does not evoke manifestations of reflex locomotion. Autonomic nervous system activity was evaluated using spectral analysis of heart rate variability before and after the intervention.

Results. The active stimulation was perceived as more unpleasant than the control stimulation. Heart rate variability parameters demonstrated almost identical autonomic responses after both stimulation types, showing either modest increase in parasympathetic activity, or increased heart rate variability with similar contribution of parasympathetic and sympathetic activity.

Conclusion. The results demonstrate changes of cardiac autonomic control in both active and control stimulation, without evidence for a significant difference between the two.

Key words: heart rate variability, spectral analysis, pressure stimulation, reflex locomotion, Vojta Therapy

Received: September 26, 2017; Accepted with revision: May 16, 2018; Available online: May 23, 2018 https://doi.org/10.5507/bp.2018.028

${ }^{a}$ Department of Physiotherapy, Faculty of Physical Culture, Palacky University Olomouc, Czech Republic ${ }^{b}$ Department of Physiotherapy, Faculty of Health Sciences, Palacky University Olomouc, Czech Republic 'KM KINEPRO PLUS s.r.o., Olomouc, Czech Republic

${ }^{d}$ Department of Neurology, Faculty of Medicine and Dentistry, Palacky University Olomouc and University Hospital Olomouc, Czech Republic Corresponding author: Pavel Hok, e-mail: pavel.hok@upol.cz

\section{INTRODUCTION}

The technique of reflex locomotion according to Václav Vojta ${ }^{1}$ belongs to neurophysiological physiotherapeutic methods, currently used in many disorders and injuries affecting the central and/or peripheral nervous or musculoskeletal systems. Application of the technique is associated not only with motor manifestations but also with autonomic nervous system (ANS) responses. Whereas the influence on human motor activity has been repeatedly studied ${ }^{2-5}$, we have not found any reports of the effect of Vojta Therapy on autonomic activity and autonomic control in the published literature. Our previous study using functional MRI of the brain demonstrated specific modulation of hand motor control in the pontomedullary reticular formation (PMRF) following the stimulation according to Vojta ${ }^{5}$. Besides motor control, the PMRF is also implicated in various aspects of autonomic control ${ }^{6}$. Therefore, we decided to study the effect of Vojta Therapy on cardiac autonomic control using spectral analysis of heart rate variability (SAHRV). Stimulation in an active (trigger) zone on the lateral heel according to Vojta was compared to stimulation outside the known active zones (control or sham stimulation) within the same young healthy participants.

\section{MATERIALS AND METHODS}

\section{Subjects}

Study participants were recruited among students of health care professions at Palacky University in Olomouc. Participants were enrolled following informed consent and after keeping a recommended regime prior to the scheduled examination. Study protocol has been approved by Ethics Committee of the University Hospital and the Faculty of Medicine and Dentistry, Palacky University Olomouc, approval number 9.4.2013.

Thirty students with no history of neurologic or psychiatric disease were included. Two participants were excluded after initial autonomic examination, one of them manifested extremely high and the other extremely low values of heart rate variability (HRV) spectral parameters, which did not permit reliable assessment of 


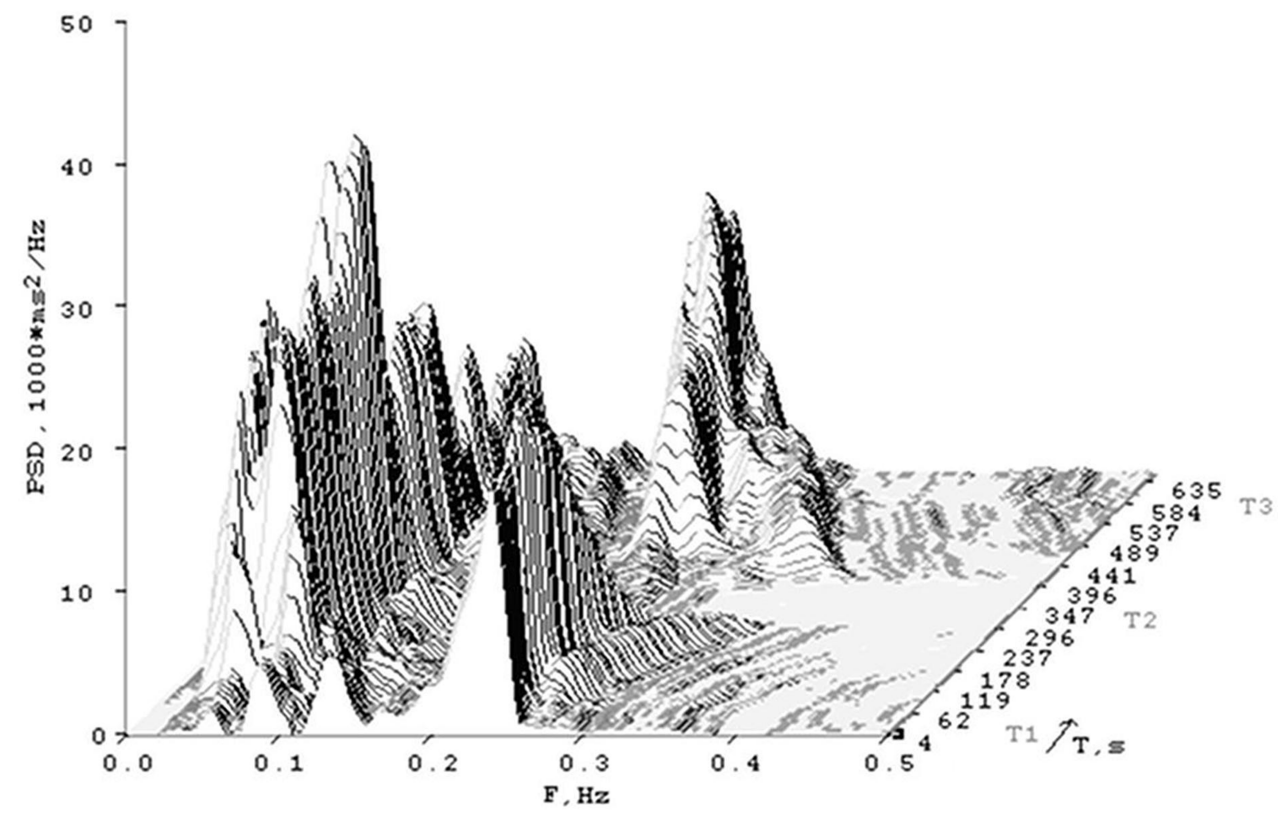

Fig. 1. Spectral analysis of heart rate variability in a young healthy subject during the supine-standing-supine test.

PSD - Power spectral density, F - frequency, T - time. T1 - first supine phase, T2 - standing phase, T3 - repeated supine phase. Frequency ranges: Low frequency (LF) $-0.05-0.15 \mathrm{~Hz}$, high frequency (HF) $0.15-0.50 \mathrm{~Hz}$. Note the clear decrease in the HF component in the standing position (T2), corresponding to decreased vagal activity, and its return to the previous level (or above that) in the repeated supine position (T3).

changes during different phases of testing. The investigated group therefore included 28 participants ( 15 women and 13 men), mean age 23.3 years, range 20.4-25.7 years.

\section{Assessment of cardiac autonomic control - spectral analysis of heart rate variability}

Cardiac autonomic control was studied on short-term ECG recordings, evaluating so-called short-term heart rate variability ${ }^{7}$. We have used a modification evaluating the orthoclinostatic reaction in the supine-standing-supine test $^{8-10}$ to be able to register the changes (shift) in cardiac autonomic control in situations with different orthostatic load. It was chosen due to the fact that vagal activity prevails in the supine body position, whereas in the standing position vagal influence on heart decreases and sympathetic activity increases. The acquired short-term ECG recordings were subjected to temporal and spectral analysis of HRV using the DiANS PF8 system (Dimea Group, Olomouc, Czech Republic). Spectral calculations were performed with fast Fourier transform using a partially modified algorithm CGSA (coarse-graining spectral analysis) (ref. ${ }^{11}$ ), with suppression of noise components.

The duration of each of the three phases of the supinestanding-supine test depended on the heart rate of each investigated individual, about $5 \mathrm{~min}$ on average. The entire supine-standing-supine test thus lasted about 15 minutes (at a heart rate of 60 beats per minute). Details of the investigation and evaluation for SAHRV examination have been published elsewhere ${ }^{9}$.

The HRV analysis yielded the following parameters in the frequency domain related to cardiac autonomic control for short-time recordings: VLF Power $\left(\mathrm{ms}^{2}\right)=$ spectral power of the very low frequency band $0.02 \mathrm{~Hz}-0.05 \mathrm{~Hz}$, LF Power $\left(\mathrm{ms}^{2}\right)=$ spectral power of the low frequency band $0.05 \mathrm{~Hz}-0.15 \mathrm{~Hz}$, HF Power $\left(\mathrm{ms}^{2}\right)=$ spectral power of the high frequency band $0.15 \mathrm{~Hz}-0.50 \mathrm{~Hz}, \mathrm{LF} / \mathrm{HF}$ ratio = ratio of spectral powers LF over HF, Relative VLF $(\%)=$ relative representation of the VLF component in the entire frequency range $(0.02-0.50 \mathrm{~Hz})$, Relative LF $(\%)=$ relative representation of the LF component in the entire frequency range, Relative $\mathrm{HF}(\%)$ = relative representation of the HF component in the entire frequency range, Total Power $\left(\mathrm{ms}^{2}\right)=$ total spectral power over the entire frequency range $0.02-0.50 \mathrm{~Hz}$. In the time domain: $\mathrm{MSSD}=$ mean squared successive differences indicator of HRV, RR interval (s) = duration of the RR interval derived from ECG. See Fig. 1 for graphical representation of the spectral analysis.

\section{Respiratory rate assessment}

Respiratory rate is another autonomic variable, which needs to be recorded and considered for an SAHRV study. Participants were breathing at their natural pace, respiration was recorded continuously with the DiANS PF8 system and simultaneously using adjustable chest belt with sensor. Respiration frequency was assessed in each of the three phases of the supine-standing supine test, together with SAHRV parameters in the same protocol.

\section{Assessment of the degree of stimulation discomfort (unpleasantness of the stimulation)}

Stimulation, which evokes unpleasant feelings, including pain, will influence and modify ANS activity. We have therefore used a visual analogue scale (VAS) to capture 


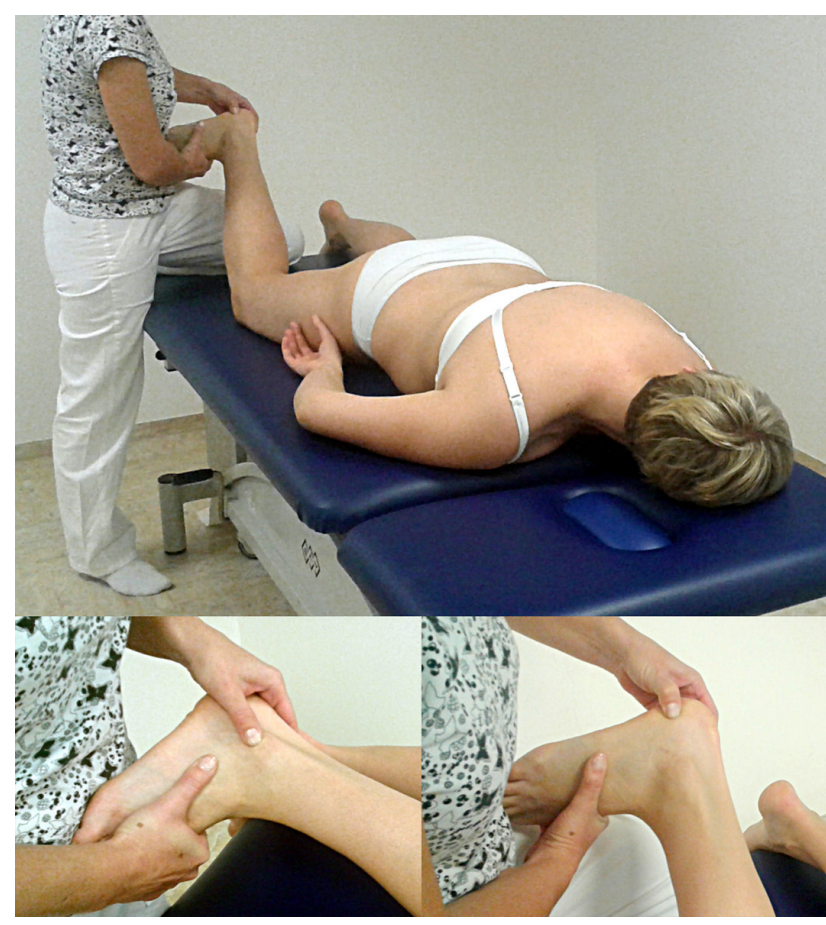

Fig. 2. Stimulation procedure and stimulation sites. The upper photograph shows the body position during the stimulation. The lower right photograph shows the stimulation site (zone) at the right lateral heel according to Vojta, whereas the lower left photograph shows the control stimulation site at the right lateral ankle.

the degree of stimulation discomfort, that is the degree of unpleasantness of the stimulation, so that we might account for the possible influence of negatively perceived stimulation or frank nociception on the SAHRV parameters, which might prevail over the effect of the two different types of manual stimulation. VAS was assessed on a scale 0 to 10 (minimum and maximum discomfort sensation, respectively), the perceived value was recorded immediately after stimulation end.

\section{Procedures}

Each participant underwent two autonomic nervous system examinations (SAHRV), at least a week apart (maximum 5 weeks). Each session was scheduled at 11 a.m. and used one or the other of two stimulation sites (see below), the order of examinations was randomized. Stimulation sites at the right leg were either 1) the foot zone according to prof. Vojta: processus lateralis tuberis calcanei (active stimulation) or 2) lateral ankle (sham stimulation), see Fig. 2. During both stimulation types, the participant lay prone, in an initial position for the so called reflex crawling ${ }^{1}$ and each stimulation involved 20 min of manual pressure applied by a trained and experienced physiotherapist.

To allow subsequent HRV analysis, the ECG recording was performed twice within each examination: before and immediately after $20 \mathrm{~min}$ of peripheral pressure stimulation.

As data from the "supine1" phase may be influenced by interfering factors both somatic and psychological (e.g., pre-examination stress, new experimental situation, white-coat syndrome, etc.), heart rate, SAHRV and respiration rate obtained during the third phase of the test, "supine 2" (supine position following orthostatic load in the prior standing), were used for statistical analysis (see also ref. ${ }^{8-10}$ ).

\section{Statistical analysis}

The acquired data were processed with the software Statistica 12 (StatSoft, Tulsa, OK, USA). For within-subject effects, the non-parametric Wilcoxon paired test was used, whereas between-session effects for the respiratory rate and the degree of stimulation-related discomfort were tested with the Mann-Whitney test.

\section{RESULTS}

\section{Spectral analysis of heart rate variability}

SAHRV of the first test phase, i.e., the first supine position (baseline), yielded in all participants spectral characteristics typical of healthy subjects of their age group ${ }^{9}$, with the possibility to distinguish individual spectral bands and with sufficiently high values of spectral power within individual frequency components to permit quantitative analysis, including assessment of the responses to changes in body position.

The values of the calculated HRV parameters before and after active (heel) stimulation are provided in Table 1. Statistical significance refers to results of the Wilcoxon paired test. Values of the assessed ECG and SAHRV parameters before and after ankle stimulation (control site) are provided in Table 2 .

The results indicate that both stimulation types, i.e., stimulation in an "active" site according to prof. Vojta (heel) and stimulation in a control "inactive" site were followed by statistically significant changes in MSSD values, duration of RR interval, and concurrently also in respiration rate. MSSD, which represents overall heart rate variability in the time domain, increased after both stimulation types. RR intervals lengthened (thus heart rate decreased) and respiration rate decreased after both active and control stimulations.

In the frequency domain, both stimulation types were associated with a statistically significant increase in VLF Power, HF Power and Total Power. LF Power increased significantly only after the active stimulation.

Neither the LF/HF ratio, nor the relative parameters of SAHRV, indicating the relative representation of individual frequency components, manifested any statistically significant changes after either stimulation type when compared to the pre-stimulation baseline.

\section{Respiratory rate}

Respiratory rate was assessed both before stimulation of each site (active versus control), as well as after stimulation. Before active (heel) stimulation, the group mean respiratory rate was 12.3 breaths $/ \mathrm{min}(\mathrm{SD}=2.61)$, whereas before the control (ankle) stimulation, the rate 
Table 1. Duration of RR intervals and parameters of heart rate variability: heel (active) stimulation site.

\begin{tabular}{llll}
\hline Parameter & $\begin{array}{l}\text { Pre-stimulation } \\
\text { Median }(\mathrm{Q} 1-\mathrm{Q} 3)\end{array}$ & $\begin{array}{l}\text { Post-stimulation } \\
\text { Median }(\mathrm{Q} 1-\mathrm{Q} 3)\end{array}$ & $\begin{array}{l}\text { Statistical } \\
\text { Significance }\end{array}$ \\
\hline RR interval $(\mathrm{s})$ & $1.00(0.92-1.15)$ & $1.08(1.00-1.20)$ & $P<0.001$ \\
VLF Power $\left(\mathrm{ms}^{2}\right)$ & $240.80(164.69-344.74)$ & $382.69(199.19-641.88)$ & $P=0.04$ \\
LF Power $\left(\mathrm{ms}^{2}\right)$ & $627.73(398.88-849.29)$ & $757.46(462.45-1373.38)$ & $P=0.01$ \\
HF Power $\left(\mathrm{ms}^{2}\right)$ & $1270.71(462.45-1373.38)$ & $2194.41(934.12-4842.71)$ & $P=0.02$ \\
LF/HF ratio & $0.419(0.173-0.951)$ & $0.409(0.163-0.767)$ & N.S. \\
Relative VLF $(\%)$ & $11.60(6.29-15.66)$ & $10.92(6.33-14.79)$ & N.S. \\
Relative LF $(\%)$ & $26.00(13.74-39.65)$ & $27.35(12.80-36.01)$ & N.S. \\
Relative HF $(\%)$ & $62.40(45.74-77.24)$ & $61.73(45.23-78.62)$ & N.S. \\
Total Power $\left(\mathrm{ms}^{2}\right)$ & $2246.04(1486.41-5140.27)$ & $4089.94(2066.12-6912.44)$ & $P=0.01$ \\
MSSD $\left(\mathrm{ms}^{2}\right)$ & $4681.60(2735.95-14112.02)$ & $8937.38(4604.74-16404.76)$ & $P<0.001$ \\
\hline
\end{tabular}

Legend: RR interval $(\mathrm{s})=$ duration of the RR interval derived from ECG, VLF Power $\left(\mathrm{ms}^{2}\right)=$ spectral power of the very low frequency band, LF Power $\left(\mathrm{ms}^{2}\right)=$ spectral power of the low frequency band, HF Power $\left(\mathrm{ms}^{2}\right)=$ spectral power of the high frequency band, LF/HF ratio $=$ ratio of the spectral powers LF over HF, Relative VLF $(\%)$ = relative representation of the VLF component within the entire frequency range, Relative $\mathrm{LF}(\%)=$ relative representation of the LF component within the entire frequency range, Relative HF $(\%)=$ relative representation of the HF component within the entire frequency range, Total Power $\left(\mathrm{ms}^{2}\right)=$ total spectral power over the entire frequency range $0.02-0.50 \mathrm{~Hz}, \mathrm{MSSD}=$ mean squared successive differences - indicator of heart rate variability in the time domain, Q1, Q3 $=1^{\text {st }}$ and $3^{\text {rd }}$ quartile.

Table 2. Duration of RR intervals and parameters of heart rate variability: ankle (control) stimulation.

\begin{tabular}{llll}
\hline Parameter & $\begin{array}{l}\text { Pre-stimulation } \\
\text { Median }(\mathrm{Q} 1-\mathrm{Q} 3)\end{array}$ & $\begin{array}{l}\text { Post-stimulation } \\
\text { Median }(\mathrm{Q} 1-\mathrm{Q} 3)\end{array}$ & $\begin{array}{l}\text { Statistical } \\
\text { Significance }\end{array}$ \\
\hline RR interval $(\mathrm{s})$ & $0.99(0.86-1.10)$ & $1.07(0.95-1.21)$ & $P<0.001$ \\
VLF Power $\left(\mathrm{ms}^{2}\right)$ & $283.48(163.63-478.29)$ & $451.86(203.26-797.65)$ & $P=0.009$ \\
LF Power $\left(\mathrm{ms}^{2}\right)$ & $701.21(306.40-975.36)$ & $750.45(287.81-1721.95)$ & N.S. \\
HF Power $\left(\mathrm{ms}^{2}\right)$ & $1405.82(720.84-3076.14)$ & $2436.48(835.47-3768.58)$ & $P=0.03$ \\
LF/HF ratio & $0.476(0.203-0.908)$ & $0.534(0.177-0.913)$ & N.S. \\
Relat. VLF $(\%)$ & $10.31(7.82-14.95)$ & $13.43(5.16-19.08)$ & N.S. \\
Relat. LF $(\%)$ & $27.42(15.93-36.10)$ & $29.86(12.09-41.26)$ & N.S. \\
Relat. HF $(\%)$ & $62.27(38.55-75.95)$ & $54.91(42.28-76.30)$ & N.S. \\
Total Power $\left(\mathrm{ms}^{2}\right)$ & $2617.85(1689.78-4043.30)$ & $3770.58(1893.02-6456.51)$ & $P=0.001$ \\
MSSD $\left(\mathrm{ms}^{2}\right)$ & $4814.96(2388.49-8435.96)$ & $8908.95(3451.13-12689.16)$ & $P<0.001$ \\
\hline
\end{tabular}

Legend: See Legend for Table 1.

was 12.9 breaths/min $(\mathrm{SD}=2.69)$; these values were not statistically significantly different.

After stimulation of the active zone (heel), respiratory rate decreased significantly to 10.9 breaths/min ( $\mathrm{SD}=2.73$ ), $P=0.003$. Similarly, after control (ankle) stimulation, respiratory rate decreased significantly to 11.3 breaths $/ \mathrm{min}(\mathrm{SD}=2.88), P=0.003$. The respiratory rates after the two stimulation types were not significantly different (Mann-Whitney test).

\section{Stimulation discomfort}

The VAS of pain indicated mean discomfort after active (heel) stimulation 3.01 (SD 1.94), range 0.2-7.4, whereas after ankle (control) stimulation the mean VAS score was 1.62 (SD 1.48), range 0.2-6.2, this difference was statistically significant $(P=0.003)$. This reveals, even in young healthy participants, a certain unpleasantness associated with pressure at the active stimulation site. Nevertheless, despite this difference in perceived discomfort, no SAHRV parameters were apparently affected since the results were similar in both stimulation types.

\section{Behavioral and motor responses to stimulation}

During stimulation of the active site (heel), 9 out of the 28 participants (32\%) manifested involuntary signs of muscle activation - fasciculations, finger movements, muscle twitches or the development of head rotation and/or deeper breathing. In contrast, three participants (10.7\%) were falling asleep.

During stimulation of the control site (ankle), slight head rotation appeared only in one participant $(3.5 \%$ of the group), and another one manifested deeper breathing. Tendency to fall asleep appeared in 3 participants (10.7\%), two of whom were also sleepy after the active stimulation.

\section{DISCUSSION}

Reflex locomotion, introduced by Václav Vojta, is based upon stimulation of so-called trigger zones on the surface of human body and has become one of rehabilitation methods preferably used in central nervous system disorders of childhood, especially cerebral palsy and cen- 
tral coordination disorder ${ }^{1,12}$. Later, it was also applied to disorders of the peripheral nervous system and to selected structural and functional disorders and injuries of the musculoskeletal system. Clinical studies of the Vojta Therapy focused on motor activity ${ }^{2-4,13-15}$.

Stimulation of the empirically discovered trigger zones according to Vojta, which evokes motor manifestations reflex locomotion, concurrently evokes responses of the autonomic nervous system as described in the method's characteristics ${ }^{1,16,17}$. Among them, the most significant are the cardiovascular responses, where vasomotor changes have been most frequently observed.

Surprisingly, our search of the medical and scientific literature did not discover any reports on observed influences on the autonomic nervous system in general, and neither on the heart rate (and specifically its variability) during application of the reflex stimulation according to Vojta. At the same time, changes in HRV have been studied and reported for many other types of surface or other somatosensory stimulation, including nociceptive ${ }^{18-22}$.

Among the many established approaches to evaluation of HRV, some of which have clinical application ${ }^{7,9,23-25}$, we have chosen the method of spectral analysis of HRV (SAHRV) in a modification with the changes of orthoclinostatic load ${ }^{8-10}$, which induce a shift in sympathovagal balance.

The reason for choosing this particular method was the possibility to record and assess the activity of vagal and sympathetic innervation, or their relative contribution, in different body positions (in the supine-standing-supine test) before and after specific active (Vojta) stimulation as well as before and after a control stimulation outside the described trigger zone(s).

The results indicate that both active and control stimulations were followed by statistically significant lengthening of RR intervals and an increase in measures of overall variability, both in the frequency (Total Power) and time (MSSD) domain. Likewise, both stimulation types were associated with a statistically significant increase in the high-frequency (HF) spectral power, which reflects vagal activity (this also corresponds to lengthening of the average RR interval). Nevertheless, the relative representation of individual spectral components (VLF, LF and HF) has remained mostly unchanged after both stimulation types, which suggests that the degree of sympathetic and vagal contribution to cardiac autonomic control remained unchanged as well. Smith et al. (ref. ${ }^{21}$ ) reported the results of another stimulation modality, repeated massage, in neonates, where assessment of HRV indicated increase in parasympathetic activity.

Somewhat surprising was our finding of decreased respiration rate after both active and control stimulations, this usually occurs in a relaxed condition. Here, however, the subjective perception of the two stimulation types differed according to the VAS scores, which revealed a higher degree of stimulation discomfort (unpleasantness) during stimulation of an active trigger zone of the Vojta Therapy. In both stimulation types, though, the VAS scores were low.
Overall, the changes in SAHRV parameters may be interpreted as similar after both stimulation types, namely, that stimulation of the active zone on the heel has not evoked a clearly different response than stimulation outside the active zone (ankle). This stands in apparent contradiction to previous experience with autonomic reflex responses during application of the Vojta Therapy in the clinical practice ${ }^{1}$.

There may be several reasons for this discrepancy. The typical target group for the Vojta technique, neonates and infants, has autonomic responses different from those of adults, one of the underlying factors may be the immaturity of the central nervous system in the children. The other obvious difference is the absence of CNS lesions in our research population, whereas in the clinical practice, the therapeutic stimulation is mostly applied to children with perinatal or prenatal brain damage. Taken together, the rather small and non-specific autonomic response to pressure stimulation of the foot in our young healthy adult participants (university students) may not be unexpected when the more prominent responses have been observed in children with CNS damage in the first months and years of life.

Furthermore, the therapeutic application in the clinical practice typically includes simultaneous stimulation in several trigger zones, whereas our protocol was simplified to using a single stimulation site (either active or control).

Another possible explanation for the similarity of autonomic responses after both stimulation types is the fact that our participants did not manifest obvious emotional reactions, whereas in children, Vojta Therapy is commonly accompanied by unpleasant feelings, often with pain, and concomitant autonomic responses. Last but not least, children manifest the tendency to escape and withdraw from unpleasant stimulation and the withdrawal or reflex motor behavior may be closely associated with the observed autonomic responses.

As mentioned above, the study involved several limitations: We have used the most accessible study population, young healthy adults, whereas more pronounced autonomic changes might be observed in children and/ or subjects with nervous system damage. The use of a single stimulation zone has also been mentioned already. A third group with no stimulation might have been useful to clarify test-retest variability. These issues may be addressed in future research.

\section{CONCLUSION}

Sustained manual pressure stimulation in an active (empirically discovered and clinically used) skin area on the foot was perceived as more unpleasant than stimulation of a nearby control site. Heart rate variability parameters reflecting cardiac autonomic control changes demonstrated almost identical autonomic responses after both stimulation types. Whereas several markers indicated modest increase in parasympathetic activity, other measures suggested increased heart rate variabil- 
ity together with joint increase in activity of both vagal (parasympathetic) and sympathetic activity, without significant change in their relative contribution to cardiac autonomic control. Therefore, in the present study, we were unable to demonstrate autonomic responses specific for the Vojta Therapy.

\section{ABBREVIATIONS}

ANS, Autonomic nervous system; CGSA, Coarsegraining spectral analysis; ECG, Electrocardiography; HF, High frequency; HRV, heart rate variability; LF, Low frequency; MSSD, Mean squared successive differences; PMRF, Pontomedullary reticular formation; SAHRV, Spectral analysis of heart rate variability; SD, Standard deviation; VAS, Visual analog scale; VLF, Very low frequency.

Acknowledgement: This work was supported by grant of the Czech Science Foundation (GACR) (grant number 14-22572S).

Author contributions: JO: study design, data interpretation, manuscript writing and literature search, critical revision of the manuscript; MS: data collection, Figures; MK: data collection; PH: study design, data interpretation, manuscript writing and literature search; PU: data collection, statistical analysis; HO: data collection, data analysis; PH: study design, data interpretation; the final version was approved by all authors.

Conflict of interest statement: The authors state that there are no conflicts of interest regarding the publication of this article.

\section{REFERENCES}

1. Vojta V, Peters A. Das Vojta-Prinzip: Muskelspiele in Reflexfortbewegung und motorischer Ontogenese. 3rd ed. Berlin: Springer; 2007.

2. Bäckström B, Dahlgren L. Vojta Self-training: Experiences of six neurologically impaired people: A qualitative study. Physiotherapy 2000;86(11):567-74.

3. Imamura S, Sakuma K, Takahashi T. Follow-up study of children with cerebral coordination disturbance (CCD, Vojta). Brain Dev 1983;5(3):311-4.

4. Kanda T, Pidcock FS, Hayakawa K, Yamori Y, Shikata Y. Motor outcome differences between two groups of children with spastic diplegia who received different intensities of early onset physiotherapy followed for 5 years. Brain Dev 2004;26(2):118-26.

5. Hok P, Opavský J, Kutín M, Tüdös Z, Kaňovský P, Hluštík P. Modulation of the sensorimotor system by sustained manual pressure stimulation. Neuroscience 2017;348:11-22.

6. Stremel RW, Waldrop TG, Richard CA, Iwamoto GA. Cardiorespiratory responses to stimulation of the nucleus reticularis gigantocellularis. Brain Res Bull 1990;24(1):1-6.

7. Task Force. Heart rate variability. Standards of measurement, physiological interpretation, and clinical use. Task Force of the European Society of Cardiology and the North American Society of Pacing and Electrophysiology. Eur Heart J 1996;17(3):354-81.

8. Opavský J, Salinger J. Examinational methods of the autonomic system function - a review for the clinical practice. Noninvasive Cardiol 1995;(4):139-53.

9. Opavský J. Autonomní nervový systém a diabetická autonomní neuropatie: Klinické aspekty a diagnostika. Praha: Galén; 2002.

10. Salinger J, Opavský J, Stejskal P, Vychodil R, Olšák S, Janura M. The evaluation of heart rate variability in physical exercise by using the telemetric variapulse TF3 system. Acta Univ Palacki Olomuc Gymnica 1998;28:13-23.

11. Yamamoto $Y$, Hughson RL. Coarse-graining spectral analysis: new method for studying heart rate variability. J Appl Physiol Bethesda Md 1985 1991;71(3):1143-50.

12. Vojta V. Early diagnosis and therapy of cerebral movement disorders in childhood. C. Reflexogenous locomotion - reflex creeping and reflex turning. $\mathrm{C} 1$. The kinesiologic content and connection with the tonic neck reflexes. Z Für Orthop Ihre Grenzgeb 1973;111(3):268-91.

13. Bauer H, Appaji G, Mundt D. VOJTA neurophysiologic therapy. Indian J Pediatr 1992;59(1):37-51.

14. Kanda T, Yuge M, Yamori Y, Suzuki J, Fukase H. Early physiotherapy in the treatment of spastic diplegia. Dev Med Child Neurol 1984;26(4):438-44.

15. Senthilkumar S, Swarnakumari P. A study on Vojta therapy approach to improve the motor development of cerebral palsy children. Int J Med Sci 2011;4(1\&2):39-45.

16. Dimitrijević $L$, Jakubi BJ. The importance of early diagnosis and early physical treatment of cerebral palsy. Facta Univ Ser Med Biol 2005;12(3):119-22.

17. Kotnik SS. Vojta in rehabilitation of children with neurodevelopmental disorders. Paediatr Croat 2012;56(Supplement 1):227-31.

18. Baker J, Shoemaker KJ. Effect of isometric handgrip exercise on heart rate variability with and without somatosensory stimulation. FASEB J 2013;27(1 Supplement):Ib833-Ib833.

19. Joseph P, Acharya UR, Poo CK, Chee J, Min LC, lyengar SS, Wei H. Effect of reflexological stimulation on heart rate variability. ITBMRBM 2004;25(1):40-5.

20. Koenig J, Jarczok MN, Ellis RJ, Hillecke TK, Thayer JF. Heart rate variability and experimentally induced pain in healthy adults: a systematic review. Eur J Pain Lond Engl 2014;18(3):301-14.

21. Smith SL, Lux R, Haley S, Slater H, Beachy J, Moyer-Mileur LJ. The effect of massage on heart rate variability in preterm infants. $J$ Perinatol 2013;33(1):59-64.

22. Wijnen VJM, Heutink M, van Boxtel GJM, Eilander HJ, de Gelder B. Autonomic reactivity to sensory stimulation is related to consciousness level after severe traumatic brain injury. Clin Neurophysiol 2006;117(8):1794-807.

23. Ernst G. Heart Rate Variability. London: Springer-Verlag; 2014.

24. Gang Y, Malik M. Heart Rate Variability Analysis in General Medicine. Indian Pacing Electrophysiol J 2003;3(1):34-40.

25. Vlčková E, Bednařík J, Buršová Š, Šajgalíková K, Mlčáková L. Spectral Analysis of Heart Rate Variability - Normative Data. Čes Slov Neurol Neurochir 2010;73/106(6):663-72. 